\title{
Isovitexin Depresses Osteoarthritis Progression via the Nrf2/NF-kB Pathway: An in vitro Study
}

This article was published in the following Dove Press journal:

Journal of Inflammation Research

\author{
Xiaofen $\mathrm{Hu}^{1,2, *}$ \\ Ruijie $\mathrm{Li}^{1,2, *}$ \\ Ming Sun ${ }^{1,2}$ \\ Ying Kong ${ }^{1,2}$ \\ Haifeng Zhu ${ }^{1,2}$ \\ Fujiang Wang' \\ Quanqing Wan (D) ${ }^{1,2}$ \\ 'The Third Affiliated Hospital of Zhejiang \\ Chinese Medicine University, Hangzhou, \\ 310005 , People's Republic of China; \\ ${ }^{2}$ Zhejiang Chinese Medical University, \\ Hangzhou, Zhejiang Province, 3I0053, \\ People's Republic of China \\ *These authors contributed equally to \\ this work
}

Correspondence: Quanqing Wan

The Third Affiliated Hospital of Zhejiang

Chinese Medicine University, 219

Moganshan Road, Hangzhou, 310005,

Zhejiang, People's Republic of China

Email13003678819@I63.com

\begin{abstract}
Purpose: Osteoarthritis (OA) is a multifactorial joint disease and inflammatory processes contribute to joint destruction. Isovitexin (IVX) is a flavone component found in passion flower, Cannabis and, and the palm that is known for its anti-inflammatory properties.

Materials and Methods: This study investigated in vitro the role and underlying mechanism used by IVX in its regulation of OA development. Effects of IVX on the viability of chondrocytes were measured by CCK- 8 assays. The phenotypes of extracellular matrix (ECM) degeneration and inflammation were measured by qPCR, Western blot, and ELISA; and NF- $\kappa$ B pathway was detected by immunofluorescence and Western blot. Molecular docking was applied to predict the interacting protein of IVX, while Nrf2 was knocked down by siRNAs to confirm its role.

Results: We demonstrated that IVX suppressed ECM degeneration and suppressed proinflammatory factors in IL-1 $\beta$-treated chondrocytes. Additionally, IVX impact on NF- $\mathrm{kB}$ signaling in IL-1 $\beta$-exposed chondrocytic cells; Mechanistically, it was also demonstrated in molecular docking and knock down studies that IVX might bind to Nrf2 to suppress NF- $\mathrm{BB}$ pathway.

Conclusion: Our data suggest that IVX halts OA disease advancement through the Nrf2/ $\mathrm{NF}-\mathrm{kB}$ axis, suggesting a possibility of IVX as a target for OA therapy.
\end{abstract}

Keywords: osteoarthritis, isovitexin, extracellular matrix, NF- $\kappa \mathrm{B}, \mathrm{Nrf2}$

\section{Introduction}

In general, Osteoarthritis (OA), is known as a chronic, incurable, as well as degenerating illness, which is marked with tremendous pain, limited mobility and disability. ${ }^{1,2}$ With a large aging population, increased incidences of obesity, and occasional injury, OA has become prevalent around the world. Thus far, around 27 million Americans and over 250 million people globally are affected by this disease. ${ }^{3,4}$ Advances in biological tissue engineering have attempted to ease treatment and rehabilitation of OA. ${ }^{5}$ But more work needs to be done to achieve success. Stem cell therapy also is lagging behind in its ability to relieve OA. ${ }^{6,7}$ Additionally, joint replacement surgery is costly. ${ }^{8}$ Given these complications, it is urgent and necessary to develop new drugs with high efficacy to relieve OA symptoms. This study, therefore, examined the role and efficacy of IVX in managing OA.

Inflammatory factors are known to control OA development. ${ }^{9}$ Among the chief cytokines, interleukin-1 $\beta$ (IL-1 $\beta$ ) was reported to be highly expressed in OA animals and humans, and the level of IL-1 $\beta$ positively correlated with the progression of disease. ${ }^{10,11}$ As expected, high IL-1 $\beta$ levels increased the production of tumor necrosis factor- $\alpha$ (TNF- $\alpha)$, interleukin-6 (IL-6), cytochrome C oxidase 
subunit 2 (COX-2), and inducible nitric oxide synthase 2 (iNOS), which severely impaired the metabolic turnover of extracellular matrix (ECM) inside knee joints. ${ }^{12,13}$ Conversely, IL-1 $\beta$ or IL-1 $\beta$ receptor inhibition were shown to have a therapeutic effect on OA. ${ }^{14,15}$

Moreover, nuclear factor kappa-light-chain-enhancer or NF- $\kappa B$ signaling modulates inflammation and IL-1 $\beta-$ mediated catabolism in chondrocytes. ${ }^{16}$ Under conditions of IL-1 $\beta$ stimulation, a phosphate group is added to the NF$\kappa \mathrm{B}$ inhibitor alpha $(\mathrm{I} \kappa \mathrm{B} \alpha)$ which becomes marked for proteasomal degradation. This allows the $\mathrm{p} 65$ protein to be liberated from its inhibitory complex and travel to the nucleus, ${ }^{16,17}$ where it activates target-specific proinflammatory or catabolic downstream genes. ${ }^{18,19}$

$\mathrm{Nrf2}$, an essential transcription factor, is primarily involved in cell homoeostasis. In fact, $\mathrm{Nrf2}$ was shown to activate antioxidant, anti-apoptotic, and anti-inflammatory pathways to maintain homeostasis. ${ }^{20}$ Multiples studies have also demonstrated an important interaction involving NF$\kappa \mathrm{B}$ and $\mathrm{Nrf2}$ needed to maintain cell homeostasis and improve inflammation. ${ }^{21,22}$ Interestingly, Nrf2 stimulation was also shown to depress the IL-1 $\beta$-mediated stimulation of NF- $\kappa \mathrm{B}$ in human chondrocytic cells. ${ }^{23}$ Furthermore, an imbalance in the levels of Nrf2 and NF- $\mathrm{KB}$ was reported to cause numerous diseases, including neurodegeneration. ${ }^{24}$ Based on these reports, the Nrf2/NF- $\mathrm{BB}$ pathway is deemed crucial for investigations into promising targets for $\mathrm{OA}$ therapy. Heme oxygenase 1 (HO-1) is considered one of the main effectors of Nrf2-dependent cell responses. ${ }^{25}$ Moreover, Nrf2/HO-1 association has been widely reported, especially in $\mathrm{OA}^{26,27}$

Isovitexin (otherwise called homovitexin or saponaretin) is a class of flavonoid, commonly found in the passion flower, Cannabis and, and the palm. ${ }^{28}$ Multiple studies have demonstrated an anti-inflammatory role of IVX. $^{29,30}$ Moreover, IVX was also reported to protect cells from inflammation via its regulation of Nrf2 signaling pathway. ${ }^{31}$ Given what is known about IVX, it is possible that its antiinflammatory action may serve as a protective measure against $\mathrm{OA}$, especially through the Nrf2/NF- $\mathrm{BB}$ pathway.

This paper aims to assess the protective aspect, if any, of IVX on OA. OA inflammation is activated via Nrf2/NF- $\mathrm{kB}$ signaling. Therefore, the IVX impact on the Nrf2/NF- $\kappa B$ axis will also be evaluated. The data presented here may aid in the advancements of future high efficacy OA therapy.

\section{Materials and Methods}

\section{Statement of Ethics}

The experimental mice are acquired from the Animal Experimental Research Center of Zhejiang Chinese Medical University (SYXK 2021-0012). The experimental scheme and ethical application are approved by the Animal Welfare and Ethics Review Committee of Zhejiang Chinese Medical University (IACUC-20,190,826-09), all operations are performed strictly in accordance with the guidelines of this Committee.

\section{Reagents and Antibodies}

Reagents used and the companies they were purchased from are listed as follows: IVX (purity $>98 \%$, Meilun Biotechnology, China and MedChemExpress LLC, USA), recombinant human IL-1 $\beta$ (PeproTech, USA), Collagenase Type II and dimethylsulfoxide (DMSO, Sigma-Aldrich, USA), Cell Counting Kit-8 (CCK-8, Dojindo, Japan). The primary $\left(1^{\circ}\right)$ antibodies used and the companies they were obtained from are listed below: against iNOS, Aggrecan, ADAMTS-5, and collagen II (COL II, Abcam, USA), against GAPDH, MMP-3, COX-2, and MMP-13 (Proteintech, China), and against IкB $\alpha, \mathrm{p}$-IкB $\alpha, \mathrm{p} 65, \mathrm{p}$-p65, Nrf2, HO1 (Cell Signaling Technology, USA). The secondary $\left(2^{\circ}\right)$ antibodies used and their companies are provided below: goat anti-mouse and anti-rabbit IgG-HRP (Bioworld, China), Alexa Fluor ${ }^{\circledR}$ 488-labelled as well as Alexa Fluor ${ }^{\circledR}$ 594-labelled goat anti-rabbit $\operatorname{IgG}(\mathrm{H}+\mathrm{L})$ (Jackson ImmunoResearch, USA). The cell culture medium came from Gibco (Grand Island, USA), and the 4',6-diamidino-2-phenylindole (DAPI) came from Beyotime (Shanghai, China).

\section{Isolation and Cultivation of Primary Mice Chondrocytic Cells}

To isolate primary mouse chondrocytic cells, cartilages of knee belonged to 30 immature $\mathrm{C} 57 \mathrm{BL} / 6$ mice were diced into cubes, approximately $1 \mathrm{~mm}^{3}$, before Wash with PBS, and digested in $0.25 \%$ type II collagenase at $37^{\circ} \mathrm{C}$ for $2 \mathrm{hr}$. The resulting isolated cells were grown in DMEM/F12 containing 10\% FBS (Gibco) and 1\% penicillin-streptomycin solution in a humidified environment at $5 \% \mathrm{CO}_{2}$ at $37^{\circ} \mathrm{C}$. The media was replaced once in 2 days and chondrocytes from the 2 nd passage were utilized for further investigation. 


\section{Experimental Design and Cell}

\section{Transfections}

The chondrocytes were seeded in a six-well plate and cultured for $24 \mathrm{hr}$ until they were $50-70 \%$ confluent. Then, the cells were administered $10 \mathrm{ng} / \mathrm{mL}$ IL-1 $\beta$, together with differing concentrations of IVX, namely $0,25,50 \mu \mathrm{g} / \mathrm{mL}$ for $24 \mathrm{hr}$. Subsequently, we employed PCR, Western blot analysis, immunofluorescence, or ELISA assays.

As for Cell transfections, the Nrf2-siRNA and ControlsiRNA (negative control) were purchased from Santa Cruz Biotechnology (Ribo, Guangzhou, China). Then, the cells were transfected with $50 \mathrm{nM}$ siRNA using Lipofectamine 2000 siRNA transfection reagent (Thermo Fisher) for $24 \mathrm{hr}$. And the validity of siRNA was verified by Western blot in Figure 1A.

\section{Cell Viability Assay}

The Cell Counting Kit-8 (CCK-8; Dojindo Co, Japan) was employed for the assessment of cellular survival. Briefly, chondrocytic cells $\left(50,000\right.$ cells $\left./ \mathrm{cm}^{2}\right)$ were grown in $96-w e l l$ plates for $24 \mathrm{hr}$ before a $24 \mathrm{hr}$ treatment with IL-1 $\beta$ and IVX, as described previously. The cells next underwent PBSwash, and exposure to $100 \mu \mathrm{L}$ of DMEM/F12 and $10 \mu \mathrm{L}$ of CCK-8, in $37^{\circ} \mathrm{C}$ for $1 \mathrm{hr}$. Lastly, absorbance at $450 \mathrm{~nm}$ was recorded by using a microplate reader (Thermo, USA).

\section{Real-Time PCR}

TRIzol (Invitrogen, USA) was employed for total RNA isolation, following manufacturer guidelines. In the next step, a total amount of 1000 ng RNA was used for reverse
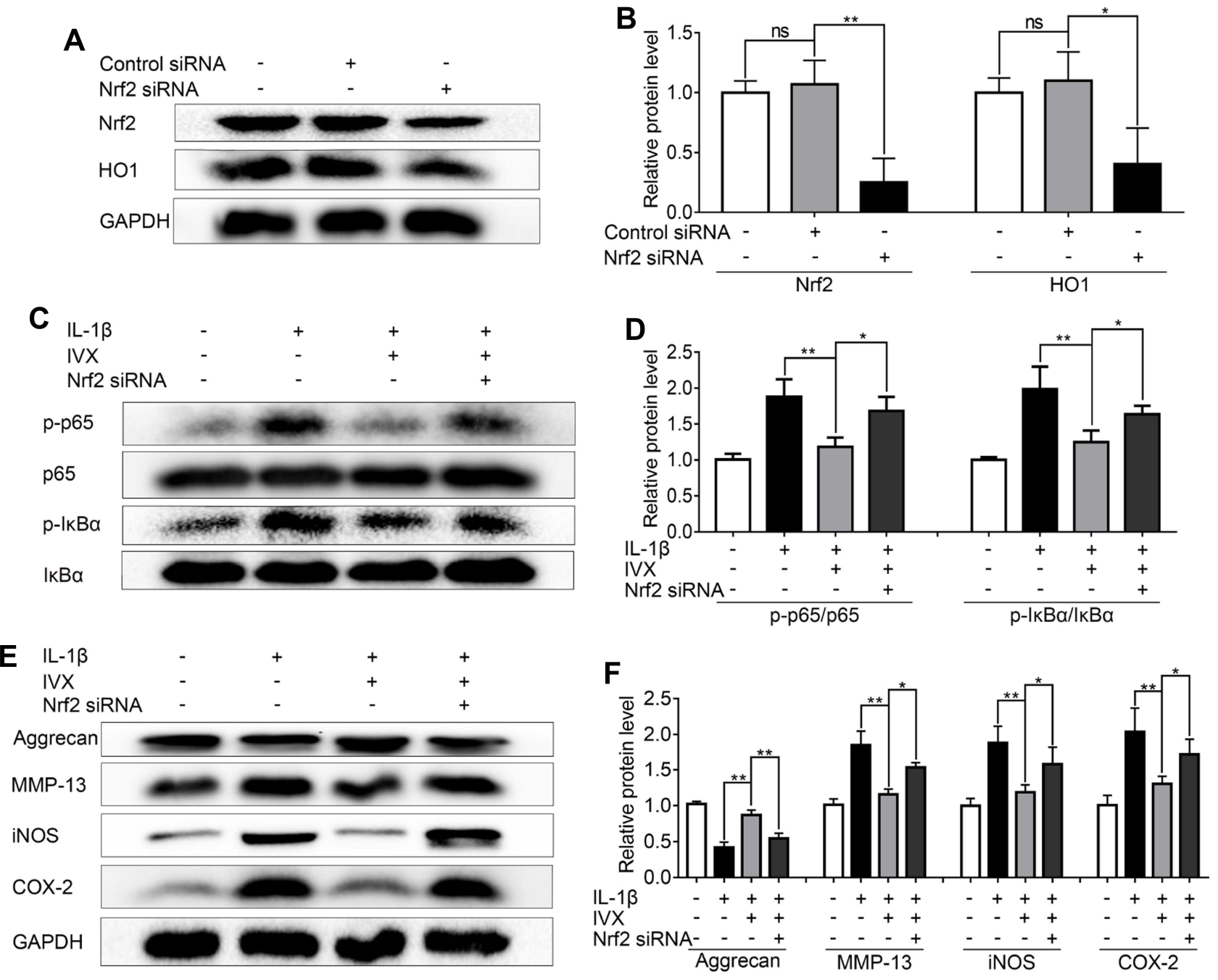

Figure I Nrf2 deficiency compromises IVX action on IL-I $\beta$-exposed chondrocytes. Nrf2 was knocked down by siRNA in IL-I $\beta$-exposed chondrocytes (A, B) Evaluation of Nrf2 and HOI protein expression, using Western blot. Protein quantification was done using Image J. (C, D) Assessment of phosphorylated and unphosphorylated forms of $1 \kappa \mathrm{B} \alpha$ and $\mathrm{p} 65$, using Western blot. Protein quantification was performed using Image J software. (E, F) Determination of the Nrf2 signaling-related protein expression. Data is average \pm SD of 3 separate examinations. ${ }^{*} p<0.05$, $* * p<0.01$.

Abbreviation: Ns, not significant. 
transcription to form cDNA (MBI Fermantas, Germany) with the kit of PrimeScript-RT reagent (Japan), as well as the System of CFX96 Real-Time PCR (Bio-Rad Laboratories, USA). cDNA amplification was done by SYBR Premix Ex Taq (Takara, Japan) in the System of CFX96 Real-Time PCR (Bio-Rad Laboratories, USA). The normalization of the cycle threshold $(\mathrm{Ct})$ of examined transcripts was carried out to the endogenous control (GAPDH). Finally, the relative levels of relevant genes were measured with the $2^{-\Delta \Delta} \mathrm{Ct}$ method. ${ }^{32}$ Lastly, the primers employed to amplify inflammatory transcripts were described previously. ${ }^{19}$

\section{Western Blot Analysis}

Chrondocyte lysis was done with radio immunoprecipitation assay (RIPA) (Beyotime, China) buffer and $1 \mathrm{mM}$ phenylmethanesulfonyl fluoride (PMSF) (Beyotime, China), before centrifugation at $4^{\circ} \mathrm{C}$ and $12,000 \mathrm{rpm}$ for $15 \mathrm{~min}$. The levels of protein were quantified using the BCA protein assessment kit (Beyotime, China). Next, $40 \mu \mathrm{g}$ of the protein was separated on an $8-12 \%(\mathrm{w} / \mathrm{v})$ sodium dodecyl sulfate polyacrylamide gel (Beijing Solarbio science and technology co., Ltd, China) electrophoresis and blotted onto the membranes of polyvinylidene fluoride (Bio-Rad, Hercules, USA). Moreover, the blocking was performed with 5\% milk with zero fat within $2 \mathrm{hr}$, then $1^{\circ}$ antibody incubation occurred at $4^{\circ} \mathrm{C}$ overnight $(\mathrm{O} / \mathrm{N})$. The $1^{\circ}$ antibodies used were against iNOS (1:1000), Aggrecan (1:800), COX-2 (1:1000), ADAMTS-5 (1:1000), p65 (1:1000), pp65 (1:800), MMP-3 (1:1000), MMP-13 (1:1000), ІкB- $\alpha$ (1:1000), p-ІкB- $\alpha$ (1:800), Nrf2 (1:1000), HO1 (1:1000), or GAPDH (1:5000). The following day, after wash 3 times with PBS, the corresponding $2^{\circ}$ antibodies were introduced for $2 \mathrm{hr}$ at room temperature (RT) before Tris-buffered saline containing Tween ${ }^{\circledR} 20$-wash $3 X$, followed by visualization with Plus reagent (Invitrogen, NY, USA) and ChemiDoc XRS+ Gel Imaging System (Bio-Rad, CA, USA). Lastly, the band intensities were meticulously explored using Image J 2.1 (Bethesda, USA), standardized by GAPDH.

\section{Immunofluorescence}

Chondrocytic cells were grown and treated in the plates including 6 wells as mentioned earlier. Next, they underwent ice-cold PBS-wash before fixation with $4 \%(\mathrm{v} / \mathrm{v})$ paraformaldehyde for 15 min, permeabilization with $0.1 \%$ Triton $\mathrm{X}-100$ diluted in PBS for $10 \mathrm{~min}$, blocked with 5\% bovine serum albumin for $1 \mathrm{hr}$ at $37^{\circ} \mathrm{C}$, exposed to $1^{\circ}$ antibodies against COL II (1:200) p65 (1:200) or $\mathrm{Nrf} 2(1: 200)$ at $4^{\circ} \mathrm{C} \mathrm{O} / \mathrm{N}$, exposed to Alexa Fluor ${ }^{\circledR} 594$-conjugated or Alexa Fluor ${ }^{\circledR} 488$ - labelled $2^{\circ}$ antibodies (1:300) for $1 \mathrm{hr}$ at RT, and stained by using 4',6-diamidino-2-phenylindole (DAPI) for $5 \mathrm{~min}$. Next, 5 random fields per slide were selected for analysis using an ECLIPSE Ti microscope of Nikon (Nikon, Japan). Lastly, scientists blinded to the sample groups were employed to quantify fluorescence intensity with Image J 2.1 (Bethesda, USA). Protein expression was quantified by integrated optical density (IOD) with the Image J 2.1 image analysis system.

\section{ELISA Assay}

Culture supernatants were collected and stored at $-20^{\circ} \mathrm{C}$ until analysis. The concentration of TNF- $\alpha$, IL- 6 , and PGE2 in cell culture supernatants was measured using ELISA kits (R\&D Systems, Minneapolis, MN, USA) according to the manufacturer's instructions.

\section{Molecular Modelling}

The IVX 3-D molecular structure was constructed utilizing Discovery Studio 2016 and minimized with the CHARMm force field. Next, the Keap1-Nrf2 structural composite (PDB ID: $3 \mathrm{WN} 7)^{33}$ was retrieved from the RCSB Protein Data Bank (https://www.rcsb.org/) and opened up in Discovery Studio 2016. Following this, the Keap1-Nrf2 composite structure was manually altered by eliminating water and introducing hydrogen before the partially flexible CDOCKER program was utilized to localize special binding locations and the receptor radius. Finally, the molecular docking data were evaluated to assess CDOCKER energy scores, interaction site, and interaction force types. ${ }^{34}$

\section{Statistical Analysis}

Data presented as average \pm SD of 3 separate examinations. Data analysis was executed with GraphPad Prism (USA) (one-way analysis of variance (ANOVA) as well as Tukey's post hoc test). Statistically significant data was given to $p$ value of $<0.05$. The variations were deemed statistically significant at $\mathrm{p}<0.05$.

\section{Results}

\section{Evaluation of IVX-Mediated Cellular} Toxicity and Protection in Chondrocytic

\section{Cells}

The IVX chemical composition is illustrated in Figure 2A. To identify if IVX exerts cytotoxicity, chondrocytic cells were given increasing concentrations of IVX, namely, 0 , $12,25,50,100,200,400 \mu \mathrm{g} / \mathrm{mL}$, for 24 or $48 \mathrm{hr}$ before cell survival measurement, as described above. Based on 
A

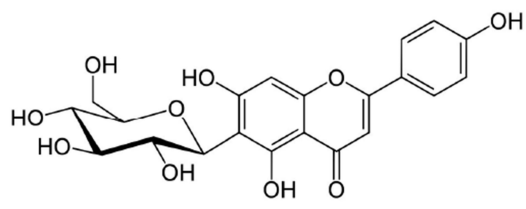

B

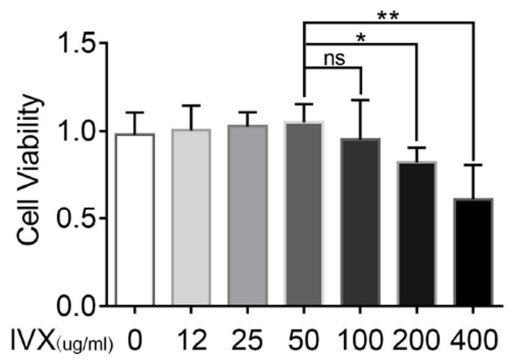

C

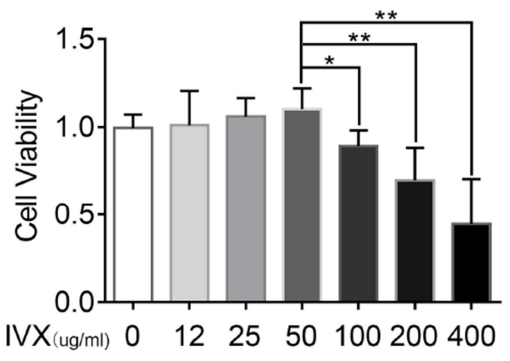

Figure 2 Evaluation of IVX-induced cellular toxicity and protection in IL-I $\beta$-exposed chondrocytic cells. (A) Chemical composition of IVX. (B, C) Assessment of IVX chondrocytic cells cytotoxicity, using differing concentrations of IVX for $24 \mathrm{hr}$ and $48 \mathrm{hr}$. Data $=$ average \pm SD of 3 separate examinations. *P $<0.05$, ** $<0.01$. Abbreviation: Ns, not significant.

our result, concentrations up to $50 \mu \mathrm{M}$ produced no observable cytotoxicity. However, IVX concentrations $>100 \mu \mathrm{M}$ markedly diminished cell survival (Figure 2B and C). Therefore, in further experimentation, IVX concentrations of 0,25 , and $50 \mu \mathrm{M}$ were used to analyze its effect on IL$1 \beta$-treated chondrocytes.

\section{IVX Impact on the Anabolic and Catabolic Nature of ECM in IL-I $\beta$ - Exposed Chondrocytes}

In order to determine whether IVX preserves ECM from IL-1 $\beta$-mediated anabolic events, anabolic proteins were evaluated in IVX- and IL-1 $\beta$-treated cells. We demonstrated a strong protection in the levels of Aggrecan and COL II in the IVX-treated cells, as opposed to cells receiving IL-1 $\beta$ alone (Figure $3 \mathrm{~A}$ and $\mathrm{E}$ ). We, further, tested IVX's effect on the ECM catabolic events and showed a strong suppression of ECM catabolic proteins, MMP-3, MMP-13, and ADAMTS-5 in the IVX-treated cells, relative to IL-alone (Figure 3A-D).

\section{IVX Modulation of Pro-Inflammatory}

\section{Factors in IL-I $\beta$-Exposed Chondrocytes}

Since pro-inflammatory factors play a major role in OA, we next examined whether IVX modulates the expression of members of the pro-inflammatory pathway. To do this, we employed PCR and ELISA assays to demonstrate significant increases in TNF- $\alpha$ and IL-6 levels upon IL-1 $\beta$ treatment, which were attenuated with exposure to IVX (Figure 4A and E). Similarly, using PCR and Western blot analysis, we revealed dramatic IL- $1 \beta$-stimulated rise in iNOS and COX-2 generation, which were suppressed in the presence of IVX (Figure 4B-F). Collectively, these results suggest that IVX represses pro-inflammatory pathways.

\section{IVX Impact on NF- $\kappa B$ Signaling in IL-I $\beta$ - Exposed Chondrocytic Cells}

Given that IVX strongly suppressed pro-inflammatory factors, we next investigated whether the cytoprotective actions of IVX involved the NF- $\mathrm{B}$ signaling pathway. Using immunofluorescence, we demonstrated p65 translocation to the nucleus in IL-1 $\beta$-exposed chondrocytic cells. Comparatively, IVX exposed cells exhibited more p65 localization in the cytoplasm (Figure 5A). Moreover, Western blot analysis revealed a larger population of phosphorylated p65 and IкB $\alpha$ in IL-1 $\beta$-exposed chondrocytic cells, as opposed to cells with no treatment, suggesting stimulation of the NF- $\kappa$ B signaling. Comparatively, the IVX-treated chondrocytes displayed remarkably low quantities of phosphorylated p65 and I $\mathrm{IB} \alpha$ (Figure 5B-D). We further isolated chondrocyte nucleus and examined p65 protein expression in the nucleus using Western blot. Similar to our immunofluorescence data, IVX suppressed p65 presence in the nucleus (Figure 5B and E). In all, these results suggest a strong $\mathrm{NF}-\kappa \mathrm{B}$ signaling repression in the IVX-exposed chondrocytic cells.

\section{Molecular Docking of IVX and Nrf2}

Multiple upstream regulators are known to modulate NF$\kappa \mathrm{B}$ signaling. Among them, Nrf2 was shown to be a major regulator of this pathway ${ }^{26}$ and serve as a potential target for OA therapy. ${ }^{35}$ To identify possible interaction between IVX and protein(s) involved with the Nrf2/NF- $\kappa \mathrm{B}$ pathway, we conducted molecular docking analysis. Our analysis predicted that the Nrf2 protein contains a binding site for IVX (Figure 6A and B). In fact, we discovered high interaction energy between IVX and Nrf2, measuring at 


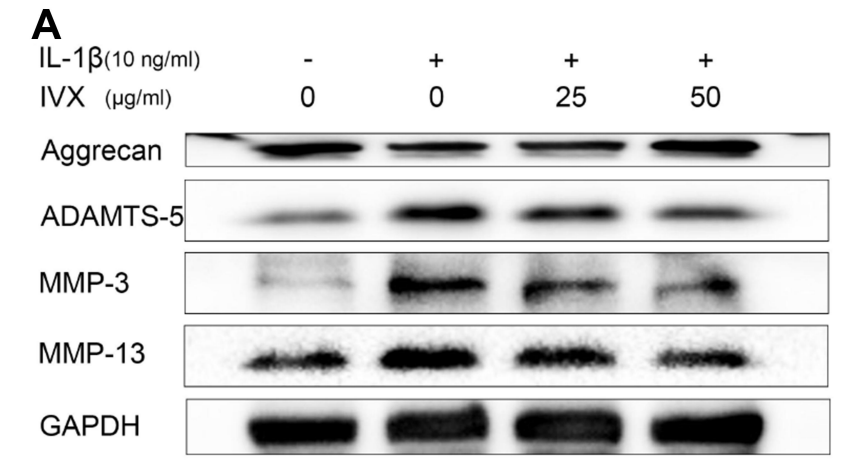

C

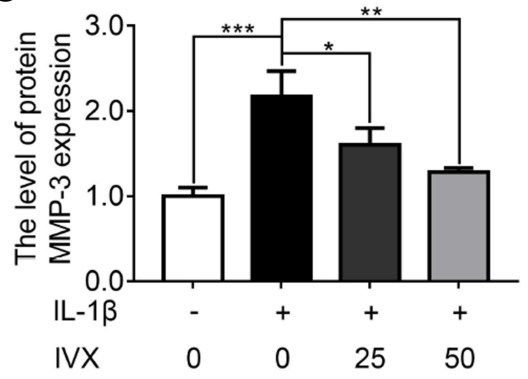

D

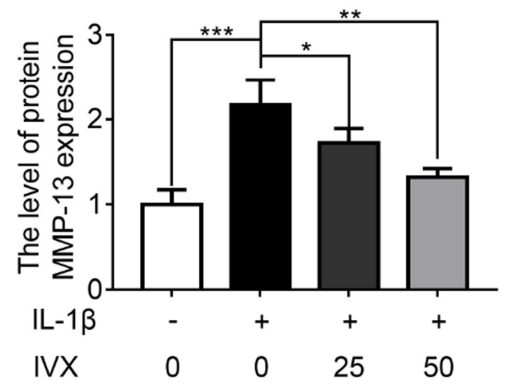

E

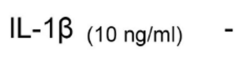

IVX $(\mu \mathrm{g} / \mathrm{ml}) \quad 0$

COL II

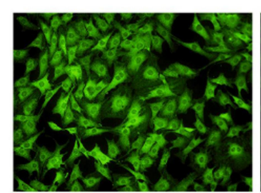

DAPI
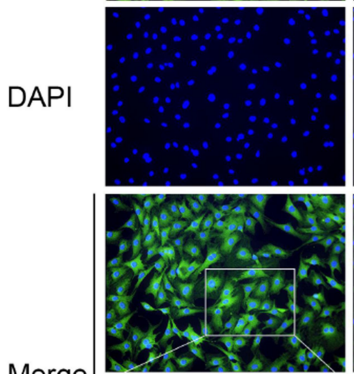

Merge

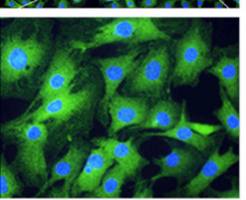

B

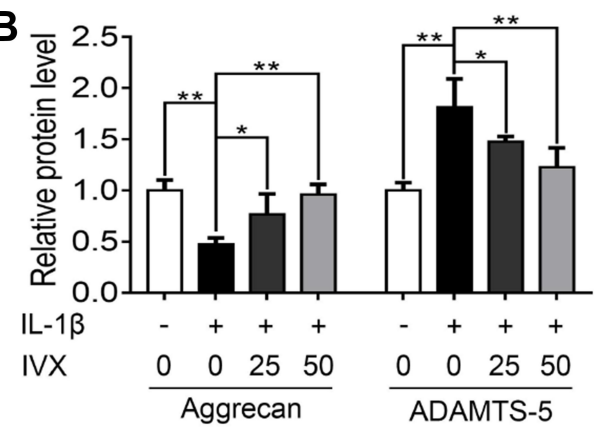

Figure 3 IVX impact on ECM anabolic and catabolic actions in IL-I $\beta$-exposed chondrocytic cells, in presence or absence of IVX. (A) Evaluation of ECM catabolic and anabolic protein expression in IL-I $\beta$ - and IVX-treated chondrocytes, relative to control. (B-D) Image J employed quantification of protein expression. (E) Detection of COL II by fluorescence microscopy, in conjunction with nuclear DAPI stain (scale bar: $50 \mu \mathrm{m}$ ). Data is average \pm SD of 3 separate examinations. *p $<0.05$, **p $<0.0$ I, *** $p<0.00$ I.

$42.95 \mathrm{kcal} / \mathrm{mol}$ (Figure 6C). Additionally, according to the 2D-binding model, Nrf2 interacted with IVX via van der Waals, hydrogen bonds, and pi-alkyl interaction (Figure $6 \mathrm{D}$ and $\mathrm{E}$ ).

\section{IVX Modulates NF- $\kappa B$ Signaling via Nrf2}

To validate the involvement of the Nrf2 pathway in the IVXmediated cytoprotection of IL- $1 \beta$-exposed chondrocytes, we first explored Nrf2 localization using immunofluorescence. We demonstrated significant Nrf2 translocation to the nucleus, upon IVX treatment (Figure 7A). Similarly, Nrf2 levels in the nuclear fractions of chondrocytes were significantly upregulated in IVX-treated chondrocytes, relative to IL-1 $\beta$ alone. Moreover, Nrf2-targeted HO1 protein expression in whole cells extracts was also elevated, relative to IL-1 $\beta$-exposed cells (Figure 7B, D and E). These data were also verified in terms of $\mathrm{HO} 1$ transcripts levels, which remained remarkably high in IVX-exposed chondrocytic cells verses IL-1 $\beta$ alone (Figure 7C). Next, we generated Nrf2 deficiency in the isolated chondrocytes, using siRNA. Using Western blot, we revealed low expression of $\mathrm{Nrf} 2$ and $\mathrm{HO} 1$ proteins upon $\mathrm{Nrf} 2$ deficiency, relative to the negative control (Figure 1A and B). Moreover, Nrf2 deficiency produced more phosphorylated forms of p65 and $\mathrm{I} \kappa \mathrm{B} \alpha$ proteins, as compared to the control (Figure 1C and D). In all, our data indicates that IVX modulates $\mathrm{NF}-\kappa \mathrm{B}$ pathway through its regulation of $\mathrm{Nrf} 2$. Furthermore, we showed that IVX exposure raised the amount of Aggrecan protein, as opposed to IL-1 $\beta$ alone (Figure 1E). Conversely, Aggrecan protein levels were strongly suppressed under conditions of $\mathrm{Nrf} 2$ deficiency, while MMP13 protein 
A

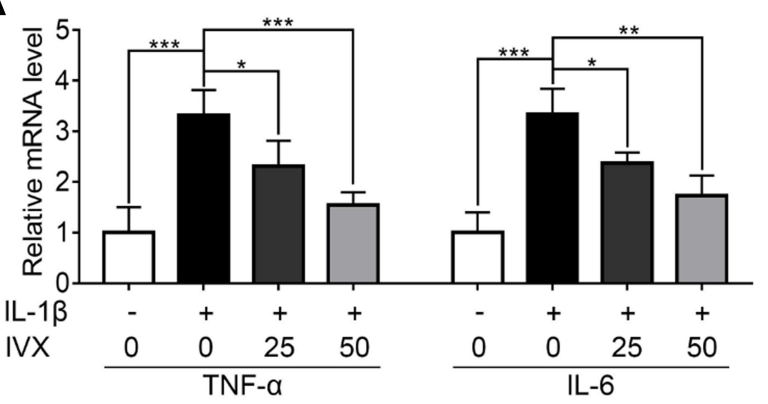

C

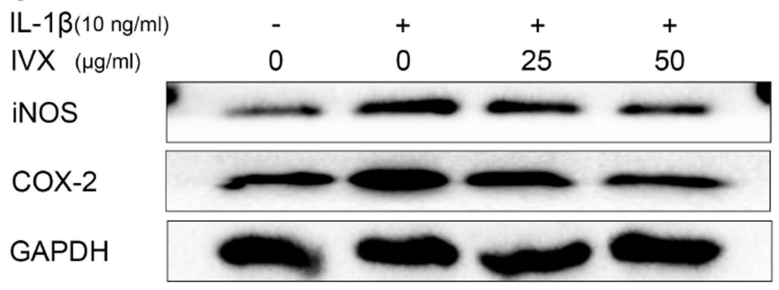

E

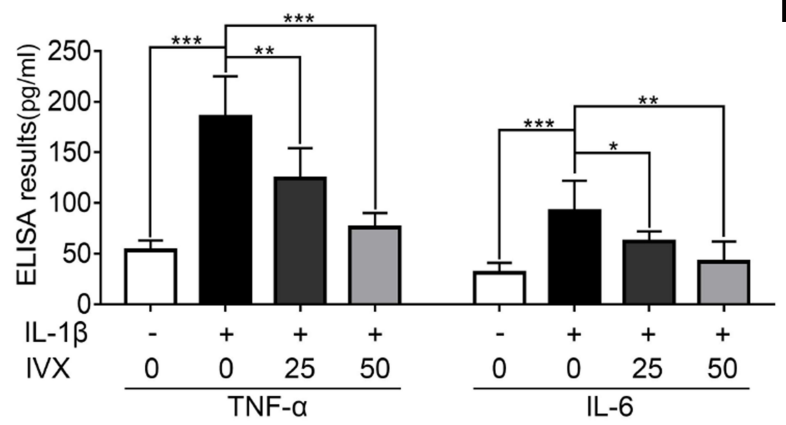

B

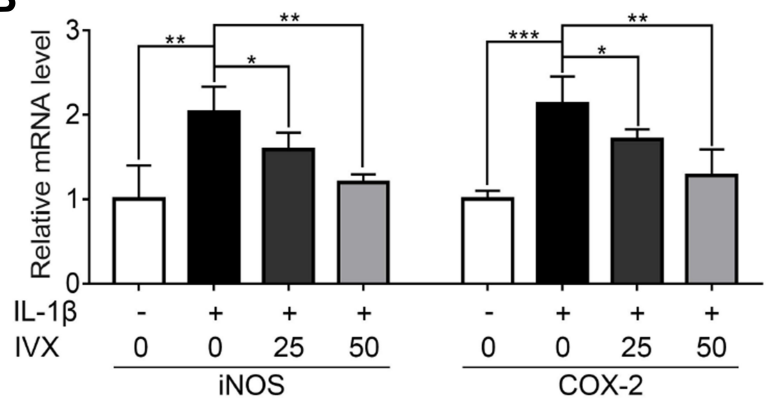

D

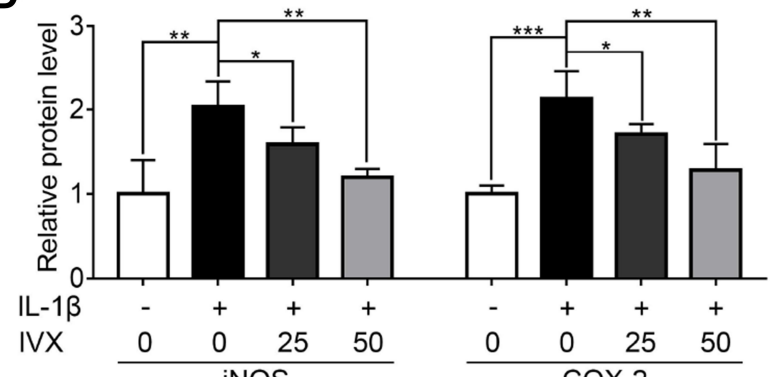

$\mathbf{F}$
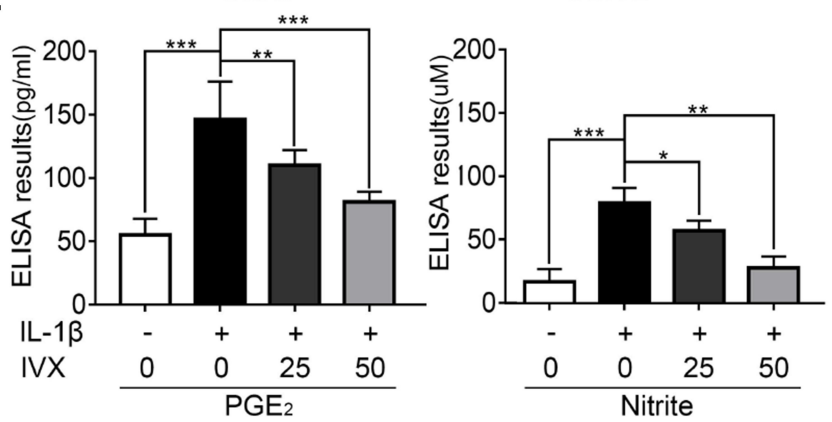

Figure 4 IVX modulation of pro-inflammatory factors in IL-I -exposed chondrocytes. (A, B) PCR analysis of inflammation-related transcripts. (C) Evaluation of inflammation-related protein expression, using Western blot. (D) Quantification of the protein expression using Image J software. (E, F) Detection of inflammationrelated protein expression, using ELISA. Data presented as average \pm SD of 3 separate examinations. $*_{p}<0.05, * * p<0.01, * * * p<0.001$.

was markedly elevated (Figure 1F). In all, these results suggest that IVX protects ECM via Nrf2 regulation. We, also, examined iNOS and COX-2 levels in the IVX-exposed chondrocytic cells, using Western blot analysis. Based on our results, IVX exposure decreased iNOS and COX-2 protein levels, whereas Nrf2 deficiency increased both protein expression (Figure 1E and F), suggesting IVX regulates inflammation via Nrf2.

\section{Discussion}

OA is a wide-spread, incurable, degenerating illness, affecting joints, and is associated with severe discomfort, limited mobility, and disability. Currently, there is no effective therapy for OA. This disease is usually characterized by severe inflammatory attack on the joints. One therapeutic agent, IVX, has shown promise in suppressing inflammation in other tissues. Therefore, this study explored whether IVX can modulate OA progression. Based on our results, we demonstrated that IVX indeed slows down OA progression by Nrf2-mediated inhibition of the NF- $\kappa \mathrm{B}$ signaling. This new finding can provide further insight into the future development of therapeutics against OA.

Multiple studies have demonstrated protective effects of IVX on inflammatory-related diseases like Alzheimer's disease, ${ }^{36}$ neuroinflammation, ${ }^{37}$ osteosarcoma, ${ }^{38}$ and liver cancer. $^{39}$ To test IVX-induced protection of OA, isolated chondrocytes were exposed to a range of working and nontoxic IVX concentrations. We confirmed that IVX was able to suppress both excessive catabolism and inadequate anabolism of ECM in IL-1 $\beta$-exposed chondrocytes. This was consistent with another study where IVX was shown to decrease matrix metalloproteinases MMP-2 expression. ${ }^{40}$ 


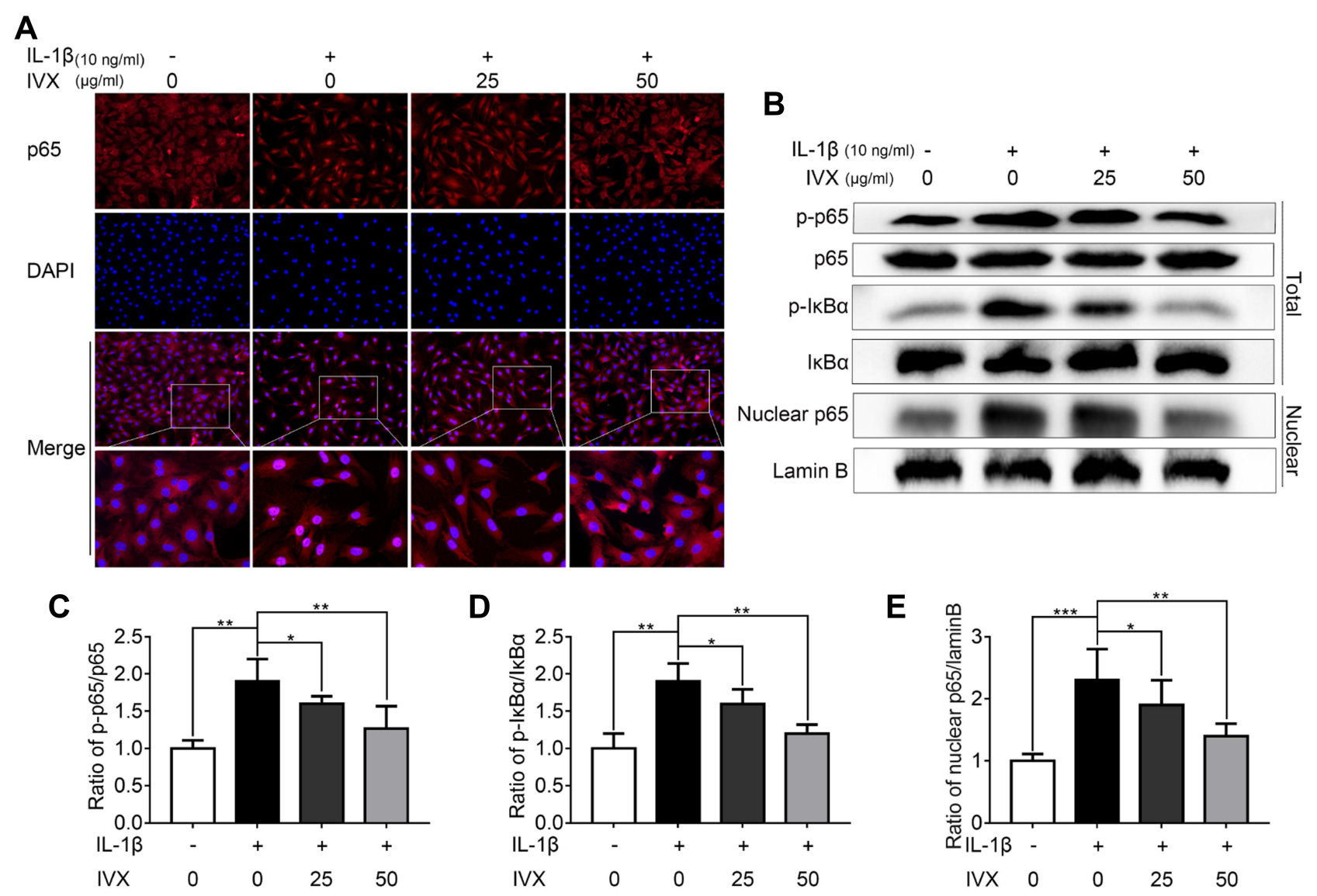

Figure 5 IVX impact on NF-kB signaling in IL-I $\beta$-exposed chondrocytic cells, with or without IVX. (A) P65 localization, detected by fluorescence microscopy, in conjunction with nuclear DAPI stain (scale bar: $50 \mu \mathrm{m}$ ). (B) Evaluation of protein expression, using Western blot. (C-E) Image J employed quantification of protein expression. Data is average $\pm S D$ of 3 separate examinations. ${ }^{*} p<0.05$, * ${ }^{*} p<0.01$, *** $<0.001$.

A

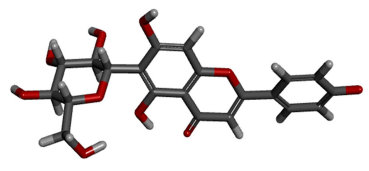

B

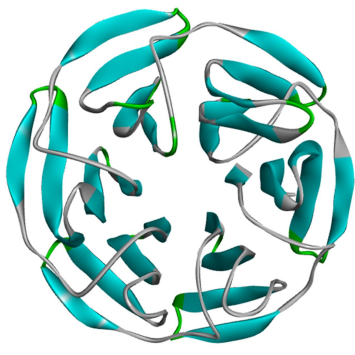

C
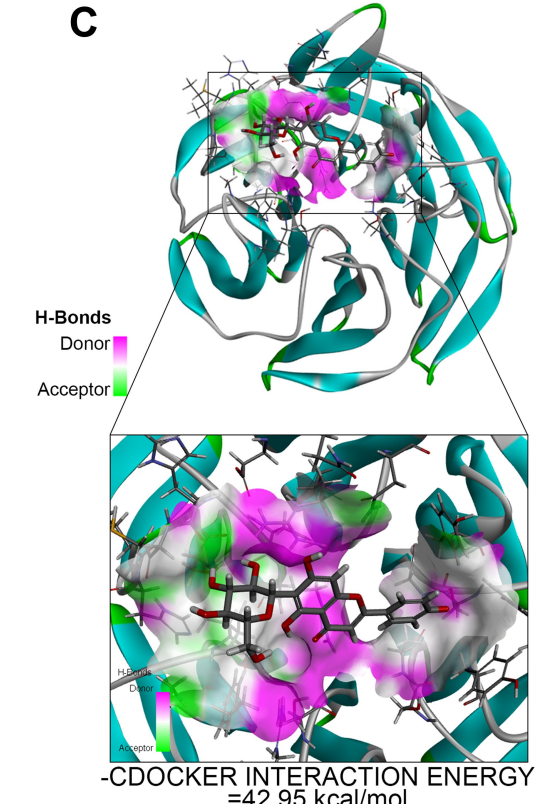

D

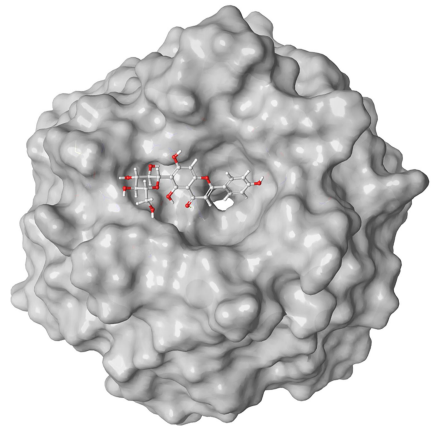

E

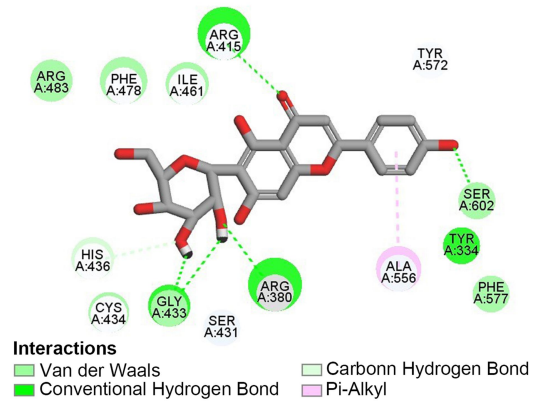

Figure 6 Molecular docking of IVX on its binding site in Nrf2, using docking studies analysis. (A) The IVX modular structure. (B) The Nrf2 ribbon modular structure. (C) The high interaction energy ( $-42.95 \mathrm{kcal} / \mathrm{mol})$ interaction between IVX and Nrf2. (D, E) The 2D binding model between IVX and Nrf2, showing interaction through van der Waals, Hydrogen Bonds, and Pi-Alkyl. 


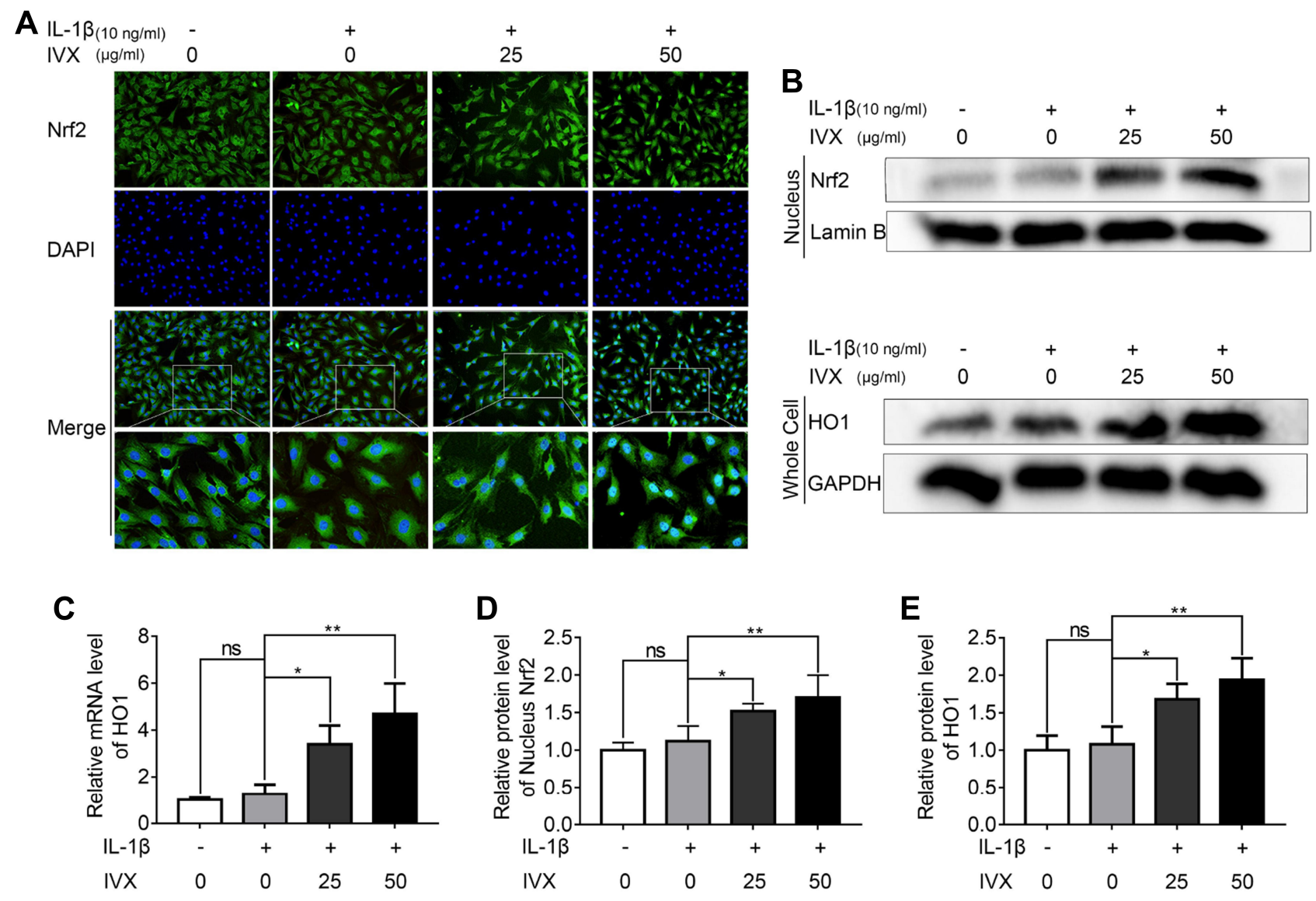

Figure 7 IVX impact on the Nrf2 signaling pathway in IL-I $\beta$-exposed chondrocytes, in the presence of or absence of IVX. (A) Detection of Nrf2, using immunofluorescence in conjunction with nuclear DAPI stain (scale bar: $50 \mu \mathrm{m}$ ). (B, D and E) Evaluation of Nrf2 levels in the nuclear fraction and downstream HOI levels in the whole extract, using Western blot. Quantification of the protein was done using Image J. (C) Evaluation of HOI transcript levels, using PCR. Data presented as average \pm SD of 3 separate examinations. ${ }^{*} \mathrm{p}<0.05$, **p $<0.01$.

Abbreviation: Ns, not significant.

Furthermore, inflammation is known to accelerate OA development, ${ }^{9}$ and existing evidences show that artificially suppressed inflammation can abrogate the progression of OA. ${ }^{27,41,42}$ In fact, Lv et al reported that IVX administration can notably reduce iNOS and COX-2 levels in monocyte/ macrophage-like cells (RAW 264.7) ${ }^{31}$ Similarly, in another study, TNF- $\alpha$ and IL-6 were strongly suppressed upon IVX treatment. ${ }^{29}$ Our findings corroborated previous studies in that IVX presence markedly lowered the amount of proinflammatory factors in IL-1 $\beta$-exposed chondrocytes.

The NF-kB pathway controls IL-1 $\beta$-mediated inflammation. ${ }^{16,43}$ In this study, IVX administration was shown to significantly suppress the NF- $\mathrm{kB}$ signaling by reducing the occurrence of phosphorylated $\mathrm{p} 65$, thereby, sequestering it in the cytoplasm. In agreement with this, earlier studies have also shown marked IVX-mediated suppression of NF- $\mathrm{kB}$ and its downstream signaling. ${ }^{30,44}$

Moreover, past studies have also demonstrated the Nrf2 signaling pathway to be a major modulator in IL-1-mediated inflammation in chondrocytic cells. ${ }^{22}$ Moreover, the NF- $\mathrm{kB}$ signaling can be effectively targeted by the Nrf2 pathway thus delaying the advancement of OA. ${ }^{26,27}$ Here, we showed strong evidence that IVX exposure can activate the Nrf2 signaling pathway in order to repress OA development. We were also able to demonstrate that IVX directly interacts with the AA residues in the inhibitory interacting pocket of Nrf2. Similar to our findings, Lv et $\mathrm{al}^{31}$ and Liu et $\mathrm{al}^{29}$ also reported that IVX can suppress Nrf2 signaling.

In conclusion, we investigated the role of IVX in modulating $\mathrm{OA}$ and explored its underlying mechanism. 


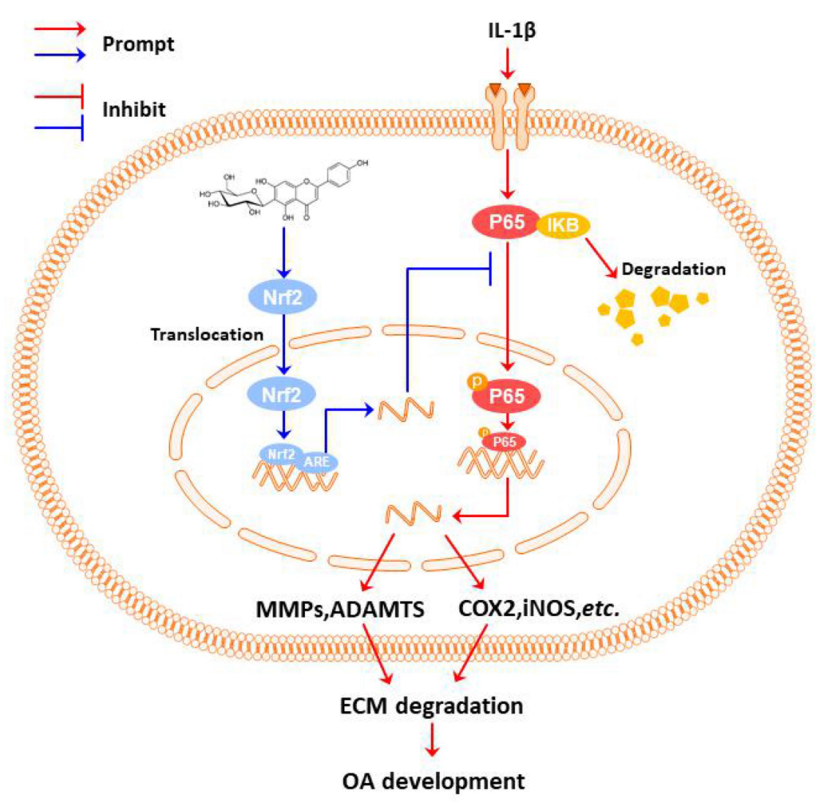

Figure 8 The proposed IVX-induced OA repression scheme. Under the stimulation of IL-I $\beta$, the chondrocytic cell undergoes inflammation and ECM degeneration; IVX can suppresses chondrocytic cell inflammation and ECM destruction via the NF- $\kappa B$ signalling pathway in its regulation of OA; Additionally, the IVX mechanism of action involved the Nrf2 activation.

We reported that IVX can indeed suppress IL-1 $\beta$-mediated inflammatory activation and maintain ECM balance in OA chondrocytic cells via the Nrf2/NF- $\kappa \mathrm{B}$ axis (Figure 8).

\section{Data Sharing Statement}

The data used to support the findings of this study are available from the corresponding author upon request.

\section{Author Contributions}

Quanqing Wan conceived of this research and helped modified the manuscript. Xiaofen $\mathrm{Hu}$ and Ruijie $\mathrm{Li}$ performed the animal model experiments and collected the samples. Haifeng Zhu and Fujiang Wang participated in the experimental design, guided the animal surgical operations, prepared reagents, materials and analytical tools. Xiaofen $\mathrm{Hu}$ wrote the manuscript. Ming Sun, Ying Kong participated in the statistical analysis, prepared all figures and embellished the manuscript. All authors made substantial contributions to conception and design, acquisition of data, or analysis and interpretation of data; took part in drafting the article or revising it critically for important intellectual content; agreed to submit to the current journal; gave final approval of the version to be published; and agree to be accountable for all aspects of the work.

\section{Funding}

This work was supported by Zhejiang Province Natural Science Foundation of China (Y17H270015, Y14H270038, LY14H270012) and Key Research projects of Science and Technology Program of traditional Chinese Medicine in Zhejiang Province(2015ZZ014).

\section{Disclosure}

The authors have no conflicts of interest.

\section{References}

1. Cross M, Smith E, Hoy D, et al. The global burden of hip and knee osteoarthritis: estimates from the global burden of disease 2010 study. Ann Rheum Dis. 2014;73(7):1323-1330. doi:10.1136/annrheumdis2013-204763

2. Storheim K, Zwart JA. Musculoskeletal disorders and the Global Burden of Disease study. Ann Rheum Dis. 2014;73(6):949-950. doi:10.1136/annrheumdis-2014-205327

3. Hunter DJ, Bierma-Zeinstra S. Osteoarthritis. Lancet. 2019;393 (10182):1745-1759. doi:10.1016/S0140-6736(19)30417-9

4. Neogi T, Zhang Y. Epidemiology of osteoarthritis. Rheum Dis Clin North Am. 2013;39(1):1-19. doi:10.1016/j.rdc.2012.10.004

5. Huey DJ, Hu JC, Athanasiou KA. Unlike bone, cartilage regeneration remains elusive. Science. 2012;338(6109):917-921. doi:10.1126/ science. 1222454

6. Djouad F, Bouffi C, Ghannam S, Noel D, Jorgensen C. Mesenchymal stem cells: innovative therapeutic tools for rheumatic diseases. Nat Rev Rheumatol. 2009;5(7):392-399. doi:10.1038/nrrheum.2009.104

7. Johnson K, Zhu S, Tremblay MS, et al. A stem cell-based approach to cartilage repair. Science. 2012;336(6082):717-721. doi:10.1126/ science. 1215157

8. Gossec L, Paternotte S, Maillefert JF, et al. The role of pain and functional impairment in the decision to recommend total joint replacement in hip and knee osteoarthritis: an international cross-sectional study of 1909 patients. Report of the OARSI-OMERACT task force on total joint replacement. Osteoarthritis Cartilage. 2011;19 (2):147-154. doi:10.1016/j.joca.2010.10.025

9. Bonnet CS, Walsh DA. Osteoarthritis, angiogenesis and inflammation. Rheumatology. 2005;44(1):7-16. doi:10.1093/rheumatology/keh344

10. Kobayashi M, Squires GR, Mousa A, et al. Role of interleukin-1 and tumor necrosis factor alpha in matrix degradation of human osteoarthritic cartilage. Arthritis Rheum. 2005;52(1):128-135. doi:10.1002/ art.20776

11. Santangelo KS, Nuovo GJ, Bertone AL. In vivo reduction or blockade of interleukin- $1 \beta$ in primary osteoarthritis influences expression of mediators implicated in pathogenesis. Osteoarthritis Cartilage. 2012;20(12):1610-1618. doi:10.1016/j.joca.2012.08.011

12. Loeser RF. Molecular mechanisms of cartilage destruction in osteoarthritis. $\quad J \quad$ Musculoskelet Neuronal Interact. 2008;8 (4):303-306.

13. Goldring MB, Otero M. Inflammation in osteoarthritis. Curr Opin Rheumatol. 2011;23(5):471-478. doi:10.1097/BOR.0b013e328349c2b1

14. Jotanovic Z, Mihelic R, Sestan B, Dembic Z. Role of interleukin-1 inhibitors in osteoarthritis: an evidence-based review. Drugs Aging. 2012;29(5):343-358. doi:10.2165/11599350-000000000-00000

15. Kerkhof HJM, Doherty M, Arden NK, et al. Large-scale meta-analysis of interleukin-1 beta and interleukin-1 receptor antagonist polymorphisms on risk of radiographic hip and knee osteoarthritis and severity of knee osteoarthritis. Osteoarthritis Cartilage. 2011;19(3):265-271. doi:10.1016/j.joca.2010.12.003 
16. Kumar A, Takada Y, Boriek AM, Aggarwal BB. Nuclear factor-kappaB: its role in health and disease. $J$ Mol Med (Berl). 2004;82(7):434-448. doi:10.1007/s00109-004-0555-y

17. Moynagh PN. The NF-kappaB pathway. J Cell Sci. 2005;118(Pt 20):4589-4592. doi:10.1242/jcs.02579

18. Zhuang Z, Ye G, Huang B. Kaempferol alleviates the interleukin-1 $\beta$ induced inflammation in rat osteoarthritis chondrocytes via suppression of NF-кB. Med Sci Monit. 2017;23:3925-3931. doi:10.12659/ MSM.902491

19. Qian Y-Q, Feng Z-H, Li X-B, et al. Downregulating PI3K/Akt/NF$\kappa \mathrm{B}$ signaling with allicin for ameliorating the progression of osteoarthritis: in vitro and vivo studies. Food Funct. 2018;9(9):4865-4875. doi:10.1039/C8FO01095A

20. Saha S, Buttari B, Panieri E, Profumo E, Saso L. An overview of Nrf2 signaling pathway and its role in inflammation. Molecules. 2020;25(22):5474.

21. Fang J, Yan Y, Teng X, et al. Melatonin prevents senescence of canine adipose-derived mesenchymal stem cells through activating NRF2 and inhibiting ER stress. Aging (Albany NY). 2018;10 (10):2954-2972. doi:10.18632/aging.101602

22. Tang S, Tang Q, Jin J, et al. Polydatin inhibits the IL-1beta-induced inflammatory response in human osteoarthritic chondrocytes by activating the Nrf2 signaling pathway and ameliorates murine osteoarthritis. Food Funct. 2018;9(3):1701-1712. doi:10.1039/ C7FO01555K

23. Kong P, Chen G, Jiang A, et al. Sesamin inhibits IL-1 $\beta$-stimulated inflammatory response in human osteoarthritis chondrocytes by activating Nrf2 signaling pathway. Oncotarget. 2016;7 (50):83720-83726. doi:10.18632/oncotarget.13360

24. Ben-Neriah Y, Karin M. Inflammation meets cancer, with NF- $\kappa$ B as the matchmaker. Nat Immunol. 2011;12(8):715-723. doi:10.1038/ ni. 2060

25. Na H-K, Surh Y-J. Oncogenic potential of Nrf2 and its principal target protein heme oxygenase-1. Free Radic Biol Med. 2014;67:353-365. doi:10.1016/j.freeradbiomed.2013.10.819

26. Shao Z-X, Wang B, Shi F-Y, et al. Senolytic agent Quercetin ameliorates intervertebral disc degeneration via the $\mathrm{Nrf} 2 / \mathrm{NF}-\kappa \mathrm{B}$ axis. Osteoarthritis Cartilage. 2021;29(3):413-422.

27. Tang Q, Feng Z-H, Tong M-J, et al. Piceatannol inhibits the IL-1betainduced inflammatory response in human osteoarthritic chondrocytes and ameliorates osteoarthritis in mice by activating Nrf2. Food Funct. 2017;8(11):3926-3937. doi:10.1039/C7FO00822H

28. He M, Min JW, Kong WL, He XH, Li JX, Peng BW. A review on the pharmacological effects of vitexin and isovitexin. Fitoterapia. 2016;115:74-85. doi:10.1016/j.fitote.2016.09.011

29. Liu S, Zhang X, Wang J. Isovitexin protects against cisplatin-induced kidney injury in mice through inhibiting inflammatory and oxidative responses. Int Immunopharmacol. 2020;83:106437. doi:10.1016/j. intimp.2020.106437

30. Lin CM, Huang ST, Liang YC, et al. Isovitexin suppresses lipopolysaccharide-mediated inducible nitric oxide synthase through inhibition of NF-kappa B in mouse macrophages. Planta Med. 2005;71(8):748-753. doi:10.1055/s-2005-871287

31. Lv H, Yu Z, Zheng Y, et al. Isovitexin exerts anti-inflammatory and anti-oxidant activities on lipopolysaccharide-induced acute lung injury by inhibiting MAPK and NF- $\kappa \mathrm{B}$ and activating $\mathrm{HO}-1 / \mathrm{Nrf} 2$ pathways. Int J Biol Sci. 2016;12(1):72-86. doi:10.7150/ijbs.13188
32. Pfaffl MW. A new mathematical model for relative quantification in real-time RT-PCR. Nucleic Acids Res. 2001;29(9):e45-e45. doi:10.1093/nar/29.9.e45

33. Tsou H, MacEwan G, Birnberg G, et al. Discovery and optimization of 2-(4-substituted-pyrrolo[2,3-b]pyridin-3-yl)methylene-4-hydroxybenzofuran-3 $(2 \mathrm{H})$-ones as potent and selective ATP-competitive inhibitors of the mammalian target of rapamycin (mTOR). Bioorg Med Chem Lett. 2010;20(7):2321-2325. doi:10.1016/j.bmcl.2010. 01.135

34. Tu M, Liu H, Zhang R, et al. Analysis and evaluation of the inhibitory mechanism of a novel angiotensin-I-converting enzyme inhibitory peptide derived from casein hydrolysate. J Agric Food Chem. 2018;66(16):4139-4144. doi:10.1021/acs.jafc.8b00732

35. Shao Z, Pan Z, Lin J, et al. S-allyl cysteine reduces osteoarthritis pathology in the tert-butyl hydroperoxide-treated chondrocytes and the destabilization of the medial meniscus model mice via the Nrf2 signaling pathway. Aging (Albany NY). 2020;12(19):19254-19272. doi:10.18632/aging.103757

36. Guimarães CC, Oliveira DD, Valdevite $\mathrm{M}$, et al. The glycosylated flavonoids vitexin, isovitexin, and quercetrin isolated from Serjania erecta Radlk (Sapindaceae) leaves protect PC12 cells against amyloid- $\beta 25-35$ peptide-induced toxicity. Food Chem Toxicol. 2015;86:88-94. doi:10.1016/j.fct.2015.09.002

37. Liu B, Huang B, Hu G, et al. Isovitexin-mediated regulation of microglial polarization in lipopolysaccharide-induced neuroinflammation via activation of the CaMKK $\beta /$ AMPK-PGC- $1 \alpha$ signaling axis. Front Immunol. 2019;10:2650. doi:10.3389/fimmu.2019. 02650

38. Liang $\mathrm{X}, \mathrm{Xu} \mathrm{C}$, Cao $\mathrm{X}$, Wang W. Isovitexin suppresses cancer stemness property and induces apoptosis of osteosarcoma cells by disruption of the DNMT1/miR-34a/Bcl-2 axis. Cancer Manag Res. 2019;11:8923-8936. doi:10.2147/CMAR.S222708

39. Lv SX, Qiao X. Isovitexin (IV) induces apoptosis and autophagy in liver cancer cells through endoplasmic reticulum stress. Biochem Biophys Res Commun. 2018;496(4):1047-1054. doi:10.1016/j. bbrc.2018.01.111

40. Qiu Y-B, Cao X-C, Liu L-H, et al. Modulation of MnSOD and FoxM1 is involved in invasion and EMT suppression by isovitexin in hepatocellular carcinoma cells. Cancer Manag Res. 2020;12:5759-5771. doi:10.2147/CMAR.S245283

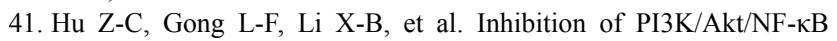
signaling with leonurine for ameliorating the progression of osteoarthritis: in vitro and in vivo studies. J Cell Physiol. 2019;234 (5):6940-6950. doi:10.1002/jcp.27437

42. Fu X, Gong L-F, Wu Y-F, et al. Urolithin A targets the PI3K/Akt/NF$\kappa \mathrm{B}$ pathways and prevents IL- $1 \beta$-induced inflammatory response in human osteoarthritis: in vitro and in vivo studies. Food Funct. 2019;10(9):6135-6146. doi:10.1039/C9FO01332F

43. Zhang C, Shao Z, Hu X, et al. Inhibition of PI3K/Akt/NF-кB signaling by aloin for ameliorating the progression of osteoarthritis: in vitro and in vivo studies. Int Immunopharmacol. 2020;89(PtB):107079. doi:10.1016/j.intimp.2020.107079

44. Hu JJ, Wang H, Pan CW, Lin MX. Isovitexin alleviates liver injury induced by lipopolysaccharide/d-galactosamine by activating Nrf2 and inhibiting NF-кB activation. Microb Pathog. 2018;119:86-92. doi:10.1016/j.micpath.2018.03.053 


\section{Publish your work in this journal}

The Journal of Inflammation Research is an international, peerreviewed open-access journal that welcomes laboratory and clinical findings on the molecular basis, cell biology and pharmacology of inflammation including original research, reviews, symposium reports, hypothesis formation and commentaries on: acute/chronic inflammation; mediators of inflammation; cellular processes; molecular mechanisms; pharmacology and novel anti-inflammatory drugs; clinical conditions involving inflammation. The manuscript management system is completely online and includes a very quick and fair peerreview system. Visit http://www.dovepress.com/testimonials.php to read real quotes from published authors. 